УДК 929

 \\ M. G. Katz ${ }^{\mathrm{g}}$, T. KudryK ${ }^{\mathrm{h}}$, S. S. Kutateladze ${ }^{\mathrm{i}}$, T. MCGaffey ${ }^{\mathrm{j}}$, \\ T. MORMAnN ${ }^{k}$, D. M. Schaps ${ }^{1}$, D. SherrY ${ }^{\mathrm{m}}$
}

\title{
CAUCHY, INFINITESIMALS AND GHOSTS OF DEPARTED QUANTIFIERS
}

\author{
Some folks see quantifiers when there are none, \\ Yet others miss infinitesimals when there are some. \\ Of history each one is a subversive brat, \\ Aping the great triumvirate, \\ While ignoring the insights of Robinson. \\ (A folk limerick)
}

J. Bair, P. Błaszczyk, R. Ely, V. Henry, V. Kanovei, K. U. Katz, M. G. Katz, T. Kudryk, S. S. Kutateladze, T. McGaffey, T. Mormann, D. M. Schaps, D. Sherry. Cauchy, infinitesimals and ghosts of departed quantifiers, Mat. Stud. 47 (2017), 115-144.

Procedures relying on infinitesimals in Leibniz, Euler and Cauchy have been interpreted in both a Weierstrassian and Robinson's frameworks. The latter provides closer proxies for the procedures of the classical masters. Thus, Leibniz's distinction between assignable and inassignable numbers finds a proxy in the distinction between standard and nonstandard numbers in Robinson's framework, while Leibniz's law of homogeneity with the implied notion of equality up to negligible terms finds a mathematical formalisation in terms of standard part. It is hard to provide parallel formalisations in a Weierstrassian framework but scholars since Ishiguro have engaged in a quest for ghosts of departed quantifiers to provide a Weierstrassian account for Leibniz's infinitesimals. Euler similarly had notions of equality up to negligible terms, of which he distinguished two types: geometric and arithmetic. Euler routinely used product decompositions into a specific infinite number of factors, and used the binomial formula with an infinite exponent. Such procedures have immediate hyperfinite analogues in Robinson's framework, while in a Weierstrassian framework they can only be reinterpreted by means of paraphrases departing significantly from Euler's own presentation. Cauchy gives lucid definitions of continuity in terms of infinitesimals that find ready formalisations in Robinson's framework but scholars working in a Weierstrassian framework bend over backwards either to claim that Cauchy was vague or to engage in a quest for ghosts of departed quantifiers in his work. Cauchy's procedures in the context of his 1853 sum theorem (for series of continuous functions) are more readily understood from the viewpoint of Robinson's framework, where one can exploit tools such as the pointwise definition of the concept of uniform convergence. As case studies, we analyze the approaches of Craig Fraser and Jesper Lützen to Cauchy's contributions to infinitesimal analysis, as well as Fraser's approach toward Leibniz's theoretical strategy in dealing with infinitesimals. The insights by philosophers Ian Hacking and others into the important roles of contextuality and contingency tend to undermine Fraser's interpretive framework.

2010 Mathematics Subject Classification: 01A45, 01A55, 01A85.

Keywords: historiography; infinitesimal; Latin model; butterfly model; law of continuity; ontology; practice; Cauchy; Leibniz.

doi:10.15330/ms.47.2.115-144

(C) J. Bair, P. Błaszczyk, R. Ely, V. Henry, V. Kanovei, K. U. Katz... , 2017 
1. Introduction. Any given period of the past was characterized by certain practices and procedures of mathematical researchers. Such practice may possess affinities with the practice of modern mathematics, whether of Weierstrassian or other variety. Practice reflects something fundamental about the subject as tackled and experienced by working mathematicians that is not always expressed in formal foundational and/or ontological work. Lurking within past practice are unexplored potentialities or possibilities. Some such possibilities did not develop fully due to contingent factors, but might have developed. Recent mathematical work can help us in doing history of mathematics by elucidating potentialities within past mathematical practice.

Our approach differs from that of Barabashev [6] who develops an absolute thesis in favor of presentism in the historiography of mathematics. We are not arguing that one should use modern mathematics to interpret the past. While we are not making any such absolute claim, the thesis we wish to develop is a relative one: to the extent that historians are already using a modern framework to interpret Leibniz, Euler, and Cauchy, namely a Weierstrassian one, we argue that a better fit for the procedures of these masters is provided by a modern infinitesimal framework. Our thesis is not that one should apriori use a modern framework, but that such a modern infinitesimal framework is preferable to the one traditionally trained historians often rely upon.

We argue, following the familiar Lakatosian dictum, that history of mathematics without philosophy of mathematics is blind. More specifically, a historiography of mathematics that fails to pay attention to the dichotomy of procedure versus ontology is flawed. Following Ian Hacking, we point out that in the historical evolution of mathematics the amount of contingency is greater than is often thought. In particular, this undermines the claim that the historical development of analysis led to modern classical Weierstrassian analysis implying that the latter must serve as a primary reference point and interpretative framework.

We compare distinct methodologies in the historiography of mathematics and point out some pitfalls inherent in neglecting the distinction of procedure versus ontology, with application to the history of infinitesimal analysis. As case studies we analyze Jesper Lützen's approach to Cauchy and Craig Fraser's approach toward both Leibniz's theoretical strategy in dealing with infinitesimals and Cauchy's contributions to infinitesimal analysis. The insights by philosophers Ian Hacking and others into the important roles of contextuality and contingency tend to undermine Lützen's and Fraser's interpretive framework.

In 2013, Bair et al. [3] presented some elements of the history of infinitesimal mathematics often unseen in the received approach to the history of analysis, based as it is upon a default Weierstrassian foundation taken as a primary point of reference. Fraser responded in 2015 with a number of criticisms in [40], but his position suffers from some of the very misconceptions analyzed in [3]. These include an insufficient attention to crucial distinctions such as practice and procedure versus ontology as developed by Benacerraf [11], Quine [80], and others. The panoramic nature of Fraser's survey enables a panoramic overview of the problematic aspects of the received historiography of mathematical analysis as it is practiced today.

We spell out some important dichotomies in Section 2. We start with an analysis of Lützen's approach in Section 3 and proceed to Fraser's starting in Section 4.

2. Dichotomies. Two dichotomies are useful in analyzing the history of analysis: Archimedean versus Bernoullian, on the one hand, and procedure versus ontology, on the other. We will analyze these dichotomies in more detail in this section. 
2.1. Parallel tracks. We propose a view of the history of analysis as evolving along separate, and sometimes competing, tracks. These are the A-track, based upon an Archimedean continuum; and B-track, based upon what could be termed a Bernoullian (i.e., infinitesimalenriched) continuum. Scholars attribute the first systematic use of infinitesimals as a foundational concept to Johann Bernoulli. While Leibniz exploited both infinitesimal methods and exhaustion methods usually interpreted in the context of an Archimedean continuum (see Bascelli et al. [7]), Bernoulli never wavered from the infinitesimal methodology. ${ }^{1}$ Historians often view the work in analysis from the 17th to the middle of the 19th century as rooted in a background notion of continuum that is not punctiform. ${ }^{2}$ This necessarily creates a tension with modern, punctiform theories of the continuum, be it the A-type set-theoretic continuum as developed by Cantor, Dedekind, Weierstrass, and others; or B-type continua as developed by Edwin Hewitt [52] in 1948, Jerzy Łoś [73] in 1955, Abraham Robinson [82] in 1966, and others. How can one escape a trap of presentism in interpreting the past from the viewpoint of set-theoretic foundations commonly accepted today, whether of type A or B?

2.2. Procedure versus ontology. A possible answer to the query formulated at the end of Section 2.1 resides in a distinction between procedure and ontology. In analyzing the work of Fermat, Leibniz, Euler, Cauchy, and other great mathematicians of the past, one must be careful to distinguish between

1. its syntactic aspects, i.e., actual mathematical practice involving procedures and inferential moves, and,

2. semantic aspects related to the justification, typically in a set-theoretic foundational framework, of entities like the points of the continuum, i.e., issues of the ontology of mathematical entities like numbers or points.

In his work on Euler and Cauchy, historian Detlef Laugwitz was careful not to attribute modern set-theoretic constructions to work dating from before the heroic 1870s, and focused instead on their procedures (concerning Laugwitz see Section 7). This theme is developed further in Section 4.3.

On the procedure/entity distinction, Abraham Robinson noted that "from a formalist point of view we may look at our theory syntactically and may consider that what we have done is to introduce new deductive procedures rather than new mathematical entities" [82, p. 282] (emphasis in the original). Kanovei et al. [57] analyze Robinson's answer to a longstanding challenge by Felix Klein and Abraham Fraenkel.

Whereas the article on Cauchy's sum theorem by Bascelli et al. [10] focuses on the details of that result of Cauchy and argues that Robinson's framework provides the best avenue for interpreting it, the present text takes a broader lens and identifies many problems in contemporary historiography of mathematics, using the work of Lützen and Fraser as examples.

3. "We miss our quantifiers". In a chapter contributed to a 2003 collection entitled $A$ History of Analysis, Lützen writes:

\footnotetext{
${ }^{1}$ To note the fact of such systematic use by Bernoulli is not to say that Bernoulli's foundation is adequate, or that it could distinguish between manipulations with infinitesimals that produce only true results and those manipulations that can yield false results.

${ }^{2}$ Historians use the term punctiform to refer to a continuum thought of as being made out of points. The term has a different meaning in modern topology that does not concern us here.
} 
(1) To the modern eye Cauchy's definitions may seem wordy, vague and not particularly rigorous. (2) We miss our quantifiers, our $\varepsilon$ 's, $\delta$ 's and $N$ 's, and in most cases our inequalities. [74, p. 161] (emphasis and sentence numbering added)

Lest a hasty reader should interpret Lützen's comment as a frank acknowledgment of the futility of a quest for ghosts of departed quantifiers in Cauchy, Lützen continues:

(3) However, as has been pointed out in particular by Grabiner ... all these ingredients are clearly present when Cauchy starts using his concepts in proofs. (4) In particular the complicated proofs are strikingly modern in appearance. (ibid.) (emphasis and sentence numbering added)

The clause "all these ingredients" in sentence (3) refers to the $\varepsilon$ 's, $\delta$ 's and $N$ 's mentioned in sentence (2). What Lützen is claiming is that the $\varepsilon$ 's, $\delta$ 's and $N$ 's appear in Cauchy's proofs, thereby making them "modern in appearance" as per sentence (4). It is worth pondering the implications of Lützen's apparent assumption that the adjective modern necessarily means $\varepsilon, \delta$. Such an assumption is indicative of a view of analysis as a teleological process with a specific implied modern outcome, which is decidedly not that of modern infinitesimals. To escape the trap of presentism, Lützen could have used the expression (in the terminology of Section 2.1) "strikingly A-track modern" ${ }^{3}$ or some such equivalent turn of phrase. But being "strikingly B-track modern" is apparently not an option in Lützen's book.

Yet Sinkevich pertinently points out that Cauchy's proofs all lack a characteristic feature of a modern epsilontic proof ("for each $\varepsilon$ there exists $\delta$, etc."), namely exhibiting an explicit functional dependence of $\delta$ on $\varepsilon$; see Sinkevich [89]. Attempts to provide A-type exhaustion justifications for results in analysis do not originate with Cauchy and go back at least to Leibniz. See e.g., Knobloch [68] for an analysis of an exhaustion procedure in Leibniz, and Blåsjö [12] for an analysis of its limitations.

3.1. Cauchy's variables. Lützen claims that "Cauchy writes that a variable having 0 as its limit becomes infinitely small" [74, p. 163]. However, Lützen's paraphrase of Cauchy's definition misstates the order of the significant clauses in Cauchy's own formulation. In fact, Cauchy's comment about the limit appears not before (as in Lützen) but after Cauchy's 1823 definition of infinitesimal:

Lorsque les valeurs numériques successives d'une même variable décroissent indéfiniment, de manière à s'abaisser au-dessous de tout nombre donné, cette variable devient ce qu'on nomme un infiniment petit ou une quantité infiniment petite. Une variable de cette espèce a zéro pour limite. ${ }^{4}[24$, p. 4] (emphasis in the original)

This indicates that limit is defined in terms of a variable quantity that becomes arbitrarily small, rather than vice versa. Indeed Cauchy wrote earlier:

Lorsque les valeurs successivement attribuées à une même variable s'approchent indéfiniment d'une valeur fixe, de manière à finir par en différer aussi peu que

\footnotetext{
${ }^{3}$ In fact Lützen appears to interpret the title of the collection his essay appeared in as referring to " $A$-History of Analysis."

4Translation: "When the successive numerical values [i.e., absolute values] of the same variable diminish indefinitely in such a way as to dip below each given number, this variable becomes what one calls an infinitely small or an infinitely small quantity. A variable of this type has limit zero."
} 
l'on voudra, cette dernière est appelée la limite de toutes les autres. ${ }^{5}[24$, p. 1]

(emphasis in the original)

The italics on limite indicate that this is the concept Cauchy is in the process of defining. Thus Cauchy defines both limits and infinitesimals in terms of the primitive notion of variable quantity, rather than using limit as a fundamental concept. Lützen goes on to claim that

[t]he standard interpretation, also adopted by Grabiner, is that the limit concept is the central one and infinitesimals only enter as useful abbreviations for variables having the limit zero [74, p. 163]

but provides no evidence in Cauchy's texts to back up his "standard interpretation" concerning limits.

3.2. Lützen versus Laugwitz-Robinson. Lützen tackles what he alleges to be a Laugwitz-Robinson interpretation of Cauchy's infinitesimals:

Laugwitz and Robinson claim that Cauchy's variables not only run through all values that correspond to our modern real numbers, but also through infinitesimals as well as sums of real numbers and infinitesimals. ... I find such a revaluation [sic] of Cauchy interesting because it highlights how historians of mathematics unconsciously read modern post-Weierstrassian ideas into Cauchy's work. [74, p. 164]

Lützen claims that Laugwitz and Robinson "unconsciously read modern post-Weierstrassian ideas into Cauchy's work." To quote a perceptive adage of the sages of the Talmud, "kol haposel, bemumo hu posel" (whoever [seeks to] disqualify [others], it is in his [own] blemish that he [seeks to] disqualify [them]). As we will see in Section 3.4, it is actually Lützen himself who reads post-Weierstrassian notions into Cauchy.

Characteristically, Lützen does not actually offer any quotes from either Laugwitz or Robinson to back up his claims. What Lützen presents as the Laugwitz-Robinson reading is the idea that Cauchy's variable quantities or magnitudes go not merely through ordinary values but also infinitesimal values.

Lützen goes on to point out that if the variable quantities already go through infinitesimal values then there would be no need to define infinitesimals afterwards as Cauchy does, since they are already in the picture: "it is hard to explain why infinitesimals are later defined afterwards as variable quantities tending to zero" [74, p. 164] (emphasis in the original) and indeed Cauchy goes on to define infinitesimals only after discussing variable quantities. QED for Lützen's refutation of Laugwitz-Robinson.

It is not an accident that Lützen fails actually to quote either Laugwitz or Robinson on this. In fact, the interpretation he seeks to refute is due to neither Laugwitz nor Robinson but rather... Fisher, who wrote in 1978:

In the Préliminaires to the Cours, Cauchy says: "When the successive numerical values of the same variable decrease indefinitely, in such a way as to fall below any given number, this variable becomes what one calls an infinitely small (un infiniment petit) or an infinitely small quantity. A variable of this kind has zero for limit" ... [37, p. 316]

${ }^{5}$ Translation: "When the values successively attributed to the same variable approach indefinitely a certain fixed value, in such a way as to differ from it by as little as one wishes, the latter is called the limit of all the others." 
Fisher concludes as follows:

The first sentence could mean that when the numerical (i.e., absolute) values of a variable decrease in such a way as to be less than any positive number, then the variable takes on infinitesimal values. (ibid.)

Lützen may be correct in questioning Fisher's interpretation of Cauchy's variables, but Lützen has not refuted either Laugwitz or Robinson whom he claims to refute, since such an interpretation is nowhere to be found in either Laugwitz or Robinson. Lützen merely introduced further confusion into the subject, and engaged in regrettable demonisation of Laugwitz and Robinson; cf. Section 7.

Lützen concludes as follows:

If the nonstandard reading of Cauchy is correct, 'magnitudes' should be ordered in a non-Archimedean way and this clashes with the Euclidean theory. [74, p. 164]

It is not entirely clear what Lützen means by "Euclidean theory." If he means to imply that no non-Archimedean phenomena can be found in The Elements, this is mostly true, but technically incorrect because we find discussions of horn angles which violate the Archimedean property when compared to rectilinear angles. Perhaps by "Euclidean theory" Lützen means Property V.4 of The Elements which is closely related to what we refer today as the Archimedean property. If so, Lützen seems to claim that Cauchy would not countenance exploiting infinitesimals which are in blatant violation of "Euclidean theory" a.k.a. Property V.4. How plausible is such a claim?

Lützen himself acknowledges that at least Cauchy's second definition of continuity does exploit infinitesimals (see Section 3.3) that evidently "clash[] with the Euclidean theory" a.k.a. V.4. Whether magnitudes and quantities go through infinitesimal values before or after Cauchy gets around to defining infinitesimals in terms thereof, there is an irreducible clash with V.4 (and of course Leibniz already pointed out that infinitesimals violate V.4; see Bascelli et al. [8], Bair et al. [4]). Lützen's allegation that this a shortcoming of what Lützen refers to as a "nonstandard reading of Cauchy" is baseless.

In the Robinson-Laugwitz interpretation, Cauchy starts with variable quantities which, as far as the 1821 book is concerned, are sequences. Cauchy says that a sequence getting smaller and smaller becomes an infinitesimal. Robinson-Laugwitz read this as saying that a sequence generates an infinitesimal which can be expressed in modern mathematical terminology by saying that infinitesimal $\alpha$ is an equivalence class of sequences, but of course Cauchy had neither sets nor equivalence classes. However, the progression of lemmas and propositions on polynomials and rational functions in $\alpha$ that Cauchy gives in the 1821 book does indicate that he is interested in the asymptotic behavior of the sequence generating an infinitesimal $\alpha$; see Borovik-Katz [20] for details. Thus, a change in finitely many terms of the sequence would not affect the asymptotic behavior. A function $f$ is applied to an infinitesimal $\alpha$ by evaluating it term-by-term on the terms of a sequence generating $\alpha$. Thus, if $\alpha$ is represented by the sequence $(1 / n)$ then the infinitesimal $\alpha^{2}$ will be incomparably smaller than alpha and will be represented by the sequence $\left(1 / n^{2}\right)$.

3.3. Lützen on Cauchy's definitions of continuity. Lützen goes on to make the following remarkable claim concerning Cauchy's definitions of continuity:

Cauchy actually gives two definitions, first one without infinitesimals and then one using infinitesimals. ... The first definition very clearly specifies a value of the variable $x$ and states that $f(x+\alpha)-f(x)$ tends to zero with $\alpha$. [74, p. 166]. 
Lützen's claim is puzzling all the more so since Cauchy's definitions are conveniently reproduced in Lützen's own text, and Cauchy clearly describes his $\alpha$ as "an infinitely small increase" [74, p. 159]. It is therefore difficult, though not impossible (see below), to maintain as Lützen does that Cauchy's first definition was "without infinitesimals." Cauchy's second definition is even more straightforward in exploiting an infinitely small increase $\alpha$ :

[Cauchy's second definition] the function $f(x)$ will remain continuous with respect to $x$ between the given limits if, between these limits an infinitely small increase [i.e., $\alpha]$ in the variable always produces an infinitely small increase in the function itself. [74, p. 160] (emphasis in the original)

The reason we wrote above that it is difficult though not impossible to maintain as Lützen does that Cauchy's first definition is "without infinitesimals" is because of the possibility of falling back on a Weierstrassian interpretation of Cauchy's infinitesimal $\alpha$ in terms of either sequences or ghosts of departed quantifiers, as other historians have done. But if it is Lützen's intention that Cauchy's $\alpha$ is only a placeholder for reassuringly Weierstrassian things, then what are we to make of Lützen's acknowledgment on page 166 (cited above) that Cauchy's second definition does exploit infinitesimals? Apparently the same infinitesimal $\alpha$ is used in both definitions, and if $\alpha$ were only a placeholder in the first definition, then $\alpha$ would be a mere placeholder in the second definition, as well. Either way you look at it, Lützen's reading of Cauchy's definitions of continuity is problematic.

At any rate we wholeheartedly agree with Lützen's assessment that Cauchy's second definition of continuity quoted above does exploit infinitesimals. If so, then Lützen would apparently have to agree with us that classical modern analysis in a Weierstrassian framework is not necessarily the best starting point for understanding at least some of Cauchy's procedures, and therefore should not necessarily serve as a primary point of reference when interpreting Cauchy's second definition of continuity, contrary to Fraser's stated position; see Sections 4 through 7.

3.4. Did Cauchy need Dedekind? In this section we examine the following startlingly ahistorical claim by Lützen:

In modern treatment [the convergence of a Cauchy sequence] is derived from the completeness of the real numbers (or is even taken as the definition of completeness) which must be either postulated as an axiom or obtained from a construction of the real numbers. Cauchy's work does not contain either way out and he could not have appealed to the underlying concept of magnitude because Euclid does not have axioms which ensure the completeness of his magnitudes.

This missing account of completeness is a fundamental lacuna which appears in several other places in Cauchy's analysis, in particular in his proof of the intermediate value theorem... [74, p. 167-168] (emphasis added)

According to Lützen, it is a "fundamental lacuna" of Cauchy's proof of intermediate value theorem that the result relies on completeness and completeness could not have been provided by Cauchy. Why is that? Says Lützen, because there are two ways of doing that: either axiomatic or construction. Lützen claims that Cauchy did not have a construction, and as far as axioms go, Euclid did not have anything on completeness.

However, Lützen'a analysis is misleading not merely because Simon Stevin already gave us unending decimals centuries earlier, but also because Laugwitz explicitly points out in his 
papers (see e.g., [71, p. 202]) that Cauchy did not need a construction of the reals because he had unending decimal expansions.

Cauchy's argument for finding a zero of a continuous function that changes sign works because it gives a procedure for finding an unending decimal expansion of such a zero, without hifalutin' embellishments concerning completeness, which is certainly a useful notion but was only introduced by Dedekind half a century later.

Thus, in the context of unending decimals Cauchy did have a suitable construction (contrary to Lützen's claim concerning an alleged fundamental lacuna) especially if one uses recursive subdivision into 10 equal parts as Stevin had done, which would directly produce an unending decimal expansion of a required zero of the function; see Błaszczyk et al. [15, Section 2.3] for details.

The Whig history aspect of Lützen's analysis is ironic since this is precisely the shortcoming he seeks to pin on Robinson and Laugwitz; see Section 3.2.

Without proposing any textual analysis of Cauchy's proof, Lützen concludes, apparently on the grounds of historical hindsight deduced from Dedekind backwards, that Cauchy's proof could not possibly be correct, because Dedekind hadn't clarified the relevant mathematical notion of completeness yet. But, as we saw, Cauchy did not need Dedekind simply because the assertion (or tacit assumption) that each unending decimal results in a legitimate real number (1) is equivalent to the completeness, and furthermore (2) could hardly be questioned by any sound pre-Weierstrassian analyst. The axiomatic formalisation of completeness was not a dramatic new discovery but rather a workhorse systematisation of common views in a new revolutionary language.

4. Role of modern theories. The relevance of modern theories to interpreting the mathematics of the past is a knotty issue that has caused much ink to be spilled. Without attempting to resolve it, we offer the following thoughts to help clarify the issue.

4.1. On the relevance of theories for history of mathematics. Let us begin by considering, as a thought experiment, the following passage.

(B) The relevance of modern Archimedean theories to an historical appreciation of the early calculus is a moot point. It is doubtful if it is possible or advisable to reconstruct a past mathematical subject in a way that conforms to modern theories and is also consistent with how a practitioner of the period would have worked. Such a project runs the obvious risk of imposing one's own interests and conceptions on the past subject. It is possible that such an endeavor will end up with something of intellectual interest and mathematical value, but it is unlikely that it will constitute a significant contribution to history.

The source of the passage (B) will be identified below. For the time being we note the following. The passage amounts to a sweeping dismissal of the modern, punctiform, Archimedean framework (as developed by Cantor, Dedekind, Weierstrass and others) as a primary point of reference for historical work dealing with prior centuries.

A reader might react with puzzlement to such a sweeping dismissal, feeling that the grounds for it are too generic to be convincing. Granted the punctiform nature of the modern continuum is different from the historical views of the continuum as found for example in Leibniz, Euler, and Cauchy. ${ }^{6}$ However, the reader may feel that the passage above ends up

${ }^{6}$ For a study of Stevin, see Katz-Katz [61] and Błaszczyk et al. [15, Section 2]; for Fermat, see Katz et al. [64] and Bair et al. [5]; for Gregory, see Bascelli et al. [9]. 
throwing out the baby with the (punctiform) bathwater.

To avoid such an overreaction one may argue as follows. For all the set-theoretic novelty of the modern view of the Archimedean continuum, surely important insight is to be gained from modern axiomatisations of the procedures in Euclid's Elements, resulting in a greater appreciation, and understanding, of this classical work (on Euclid see further in Section 5).

Similarly, historians have often endeavored to show how the lucidity of the modern Archimedean limit concept serves as a benchmark that allows us to appreciate the progress being made over the decades and centuries in clarifying the nature of the procedures involving this concept that were employed in the 17th through the 19th centuries in solving problems of analysis.

To save the baby, the reader may feel that a distinction needs to be made between, on the one hand, the modern set-theoretic justification of the entities involved, such as that of the complete Archimedean ordered field; and, on the other, the procedures and inferential moves exploited in mathematical arguments, as already discussed in Section 2.2. The former (settheoretic justification) pertains to the domain of the ontology of mathematical entities that apparently had little historical counterpart prior to 1870. Meanwhile, the latter (procedures) are, on the contrary, indispensable tools that allow us to clarify and appreciate the inferential moves as found in the work of the mathematicians of the past.

The distinction between procedure and ontology is a tool implicitly used in [40] (see Section 1). Similarly, Ferraro and Panza, both shortlisted in [40, p. 27] among the authorities on the 18th century, declare a failure of Lagrange's program of founding analysis on power series in the following terms:

[Lagrange's books] are part of a foundationalist agenda. The fact that this agenda was never really accepted by Lagrange's contemporaries contrasts with another fact: that it was the most careful attempt to integrate the calculus within the program of 18th-century algebraic analysis. Its failure is then also the failure of this ambitious program. [36, p. 189, Conclusions] (emphasis added)

One might ask, in what sense did Lagrange's program fail, if not in the modern sense of being unable to account for non-analytic functions? When restricted to analytic ones, Lagrange's program indeed succeeds. Ferraro and Panza focus on Lagrange's purity of the method but ultimately use a modern yardstick to offer an evaluation (namely, an alleged failure) of Lagrange's program, without necessarily falling into a presentist trap of attributing a punctiform continuum to Lagrange.

We hasten to add that the passage (B) cited at the beginning of the current Section 4.1 is Fraser's only up to a single, but very significant, modification. We changed his adjective non-Archimedean to Archimedean. Fraser's original passage reads as follows.

4.2. Fraser's original passage. (A) "The relevance of modern non-Archimedean theories to an historical appreciation of the early calculus is a moot point. It is doubtful if it is possible or advisable to reconstruct a past mathematical subject in a way that conforms to modern theories and is also consistent with how a practitioner of the period would have worked. Such a project runs the obvious risk of imposing one's own interests and conceptions on the past subject. It is possible that such an endeavor will end up with something of intellectual interest and mathematical value, but it is unlikely that it will constitute a significant contribution to history." [40, p. 43].

4.3. Analysis of Fraser's original. Fraser's original passage (A) appearing in Section 1 
amounts to a sweeping dismissal of the modern non-Archimedean framework as developed by Robinson, Laugwitz, and others as a primary point of historical reference.

Again, the reader might react with puzzlement to such a sweeping dismissal on what appear to be excessively generic grounds. The reader might object to Fraser's throwing out the baby with the (set-theoretic) bathwater when he ignores the distinction between modern foundational justification and classical procedures.

The upshot of our thought experiment should be clear by now. For Fraser, neither the modern Archimedean nor the modern non-Archimedean theories should be relevant to a historical appreciation of the early calculus. Rather, for him, history of mathematics stands alone and aloof. As a result it risks becoming, one may say, a vain (in both senses) exercise of "l'Histoire, c'est Moi" type; see Katz [62].

In his passage (A), Fraser implicitly argues in favor of a splendid isolation for a historiography of mathematics; according to him, it is to be pursued independently of any influence coming from modern mathematical theories whatsoever, whether of the Archimedean or the Bernoullian variety. This naturally extends to a claim that history of mathematics should be pursued independently of any theoretical modern influences whatsoever: after all, modern mathematics comes along with, like it or not, a lot of ideological baggage concerning problems of what mathematics is, how it is related to other sciences, etc.

Yet the contention that one's own conception of the history of mathematics would be free of any such baggage is hardly convincing (see especially Fraser's remarks on classical analysis as a "primary point of reference" analyzed in Section 4.6). Thus, replacing the somewhat unfriendly expression ideological baggage by the more neutral term philosophy we may assert that Fraser's intended isolationism amounts to a history of mathematics without philosophy of mathematics.

While we cautiously subscribe to Lakatos's dictum concerning the dependence of history of science on philosophy of science, ${ }^{7}$ we don't subscribe to the way in which he applied it to the history of the early calculus, preferring Laugwitz's analysis as developed in the pages of Historia Mathematica [70], Archive for History of Exact Sciences [71], and elsewhere.

As philosopher Marx Wartofsky pointed out in his programmatic contribution The Relation between Philosophy of Science and History of Science [102], there are many distinct possible relations between philosophy of science and history of science, some "more agreeable" and fruitful than others (ibid., p. 719ff). We need not go into the details of Wartofsky's typology of possible relations between the two disciplines. It will suffice to point out that according to him a fruitful relation between history and philosophy of science requires a rich and complex ontology of that science.

In the case of mathematics, this means that a fruitful relation between history and philosophy must go beyond offering an ontology of the domain over which a certain piece of mathematics ranges (say, numbers, functions, sets, infinitesimals, structures, or whatever). Namely, it must develop the ontology of mathematics as a scientific theory itself (ibid., p. 723).

In the present article, we make a step in this direction by distinguishing between the (historically relative) ontology of the mathematical objects in a certain historical setting, and its procedures, particularly emphasizing the different roles these components play in the history of mathematics. More precisely, our procedures serve as a representative of what

\footnotetext{
${ }^{7}$ Lakatos's insight as formulated in [69] was grounded upon earlier historical-philosophical studies such as Hesse [51].
} 
Wartofsky called the praxis characteristic of the mathematics of a certain time period, and our ontology takes care of the mathematical objects recognized at that time.

The urgency of drawing attention away from mathematical foundations (in a narrow ontological sense) and toward practice was expressed by Lawvere:

In my own education I was fortunate to have two teachers who used the term 'foundations' in a common-sense way (rather than in the speculative way of the Bolzano-Frege-Peano-Russell tradition). This way is exemplified by their work in Foundations of Algebraic Topology, published in 1952 by Eilenberg (with Steenrod), and The Mechanical Foundations of Elasticity and Fluid Mechanics, published in the same year by Truesdell. The orientation of these works seemed to be 'concentrate the essence of practice and in turn use the result to guide practice.' [72, p. 213]

Related educational issues were analyzed by Katz-Polev [63].

Our thought experiment in Section 2.1 comparing the passages (B) and (A) helps highlight the problem with Fraser's argument. Fraser attempts to argue against using modern Bernoullian frameworks (see Section 2.1) to interpret the mathematics of the past, but fails to take into account the crucial distinction between procedure and ontology. Therefore his argument in favor of dismissing modern frameworks is so general as to apply to his own work, and indeed to much valuable work on understanding the mathematics of the past, and has the effect of so to speak throwing out the (procedural) baby with the (ontological) bathwater. He wishes to reject applications of Bernoullian frameworks to procedures of the mathematics of the past while ignoring much of the evidence, and on such generic grounds as to make his critique untenable.

4.4. A conceptual gulf. A failure to keep in mind crucial distinctions such as that between procedure and ontology undermines Fraser's evaluation of Robinson's historical work. Writes Fraser:

The transition from algebraic analysis of the eighteenth century to Cauchy-Weierstrass analysis of the nineteenth century marked a much greater discontinuity than did the emergence of nonstandard analysis out of classical analysis in the second half of the twentieth century. [40, p. 42]

This is a valid remark (particularly if one omits the mention of Cauchy) on the nature of post-Weierstrassian analysis, and one consistent with our comments on the ontology of punctiform continua in Section 2. Fraser continues:

Nonstandard analysis is an offshoot of modern analysis and sits solidly on the modern side of the conceptual gulf opened up by the Cauchy-Weierstrass foundation. (ibid.)

Fraser again makes a valid point (again modulo Cauchy's role) concerning the ontology underwriting mathematical entities since Weierstrass. But then Fraser makes the following leap:

In this respect Robinson and to a lesser degree Lakatos were mistaken in their assessment of Cauchy. (ibid.)

This remark of Fraser's is a non-sequitur exacerbated by Fraser's failure to make explicit the alleged mistakes of Robinson and Lakatos. The following comment by Robinson already quoted in Section 2.2 may help set the record straight: 
... from a formalist point of view we may look at our theory syntactically and may consider that what we have done is to introduce new deductive procedures rather than new mathematical entities. [82, p. 282] (emphasis in the original)

Nor does Fraser provide any detailed examination of Robinson's analysis of Cauchyan texts (though a brief summary appears in [40, p. 26]). In what respect exactly is Robinson allegedly "mistaken in [his] assessment of Cauchy" as Fraser claims?

Following on the heels of Fraser's comments on the conceptual gulf wrought by settheoretic foundations that emerged following the work of Weierstrass, Fraser's remark derives its impetus from the modern nature of Robinson's framework based as it is on punctiform models of continua, contrary to pre-1870 work which was generally not based on such ontology. Yet Fraser fails to take into account the fact that in discussing Cauchy, Robinson is talking procedure, not ontology. ${ }^{8}$

4.5. Who took Cauchy out of his historical milieu? Having summarily dismissed Robinson, Fraser goes on to yet another non-sequitur:

Rather than try to understand Cauchy as someone who developed within a given intellectual and historical milieu, they approach the history from an essentially artificial point of view. [40, p. 42]

Fraser provides no evidence for his claim that Robinson seeks to take Cauchy out of his historical milieu. One may well wonder whether, on the contrary, it is Fraser who seeks to take Cauchy out of his historical milieu. Indeed, Robinson takes Cauchy's infinitely small at (procedural) face value based on an assumption that Cauchy understood the term in a sense common among his contemporaries like Abel and Poisson, as well as a majority of his colleagues at the Ecole Polytechnique, both mathematicians and physicists, whose work was a natural habitat for infinitesimals, as documented by Gilain [42].

Meanwhile, by postulating a so-called Cauchy-Weierstrass foundation (see Section 1), Fraser precisely yanks Cauchy right out of his historical milieu, and inserts him in the heroic 1870s alongside C. Boyer's great triumvirate. ${ }^{9}$

Robinson was one of the first to express the sentiment that the A-framework is inadequate to account for Cauchy's infinitesimal mathematics. Grattan-Guinness points out that Cauchy's proof of the sum theorem is difficult to interpret in an Archimedean framework, due to Cauchy's use of infinitesimals. Thus, Grattan-Guinness wrote:

This remark [of Cauchy's] is difficult to interpret against [i.e., in the context of] the classification of modes of uniform convergence given here ... since $\alpha$ is an infinitesimally small increment of $x$. [45, p. 122] (emphasis added)

Recent studies by Nakane [77] and Sørensen [93] have emphasized the difference between Cauchyan and Weierstrassian notions of limit.

Meanwhile, a B-track framework enables a better understanding of Cauchy's procedures in his 1853 text on the convergence of series of functions [28] (see Bascelli et al. [10] for

\footnotetext{
${ }^{8}$ It is worth mentioning that, among other sources, Robinson's work grew from a reflection upon Skolem's nonstandard models of arithmetic. These were developed in [90], [91], [92]. Skolem represents his nonstandard numbers by definable sequences of integers (the standard numbers being represented by constant sequences). A sequence that tends to infinity generates an infinite number. This procedure bears analogy to Cauchy's representation of the B-continuum. Indeed, Cauchy gives an example of a variable quantity as a sequence at the start of his Cours d'Analyse [23].

${ }^{9}$ Historian Carl Boyer described Cantor, Dedekind, and Weierstrass as the great triumvirate in [22, p. 298]. The term serves as a humorous characterisation of both A-track scholars and their objects of adulation.
} 
a detailed analysis) and other texts where infinitesimals are used in an essential fashion, such as Cauchy's seminal 1832 text on geometric probability [26] and his seminal 1823 text on the theory of elasticity [25]. Fraser's misjudgment of Cauchy's historical role is a predictable consequence of his dogmatic endorsement of A-track analysis as the primary point of reference for understanding the mathematics of the past, as we discuss in Section 4.6.

4.6. The apple of discord. Fraser seeks to distance himself from Boyer's view of the history of analysis as inevitable progress toward arithmetisation:

Since the 1960s there has been a new wave of writing about the history of eighteenth-century mathematics [that has] charted the development of calculus without interpreting this development as a first stage in the inevitable evolution of an arithmetic foundation. (Fraser [40, p. 27])

However, the following passage from Fraser's survey reveals the nature of his misconception:

Of course, classical analysis developed out of the older subject and it remains a primary point of reference for understanding the eighteenth-century theories. By contrast, nonstandard analysis and other non-Archimedean versions of calculus emerged only fairly recently in somewhat abstruse mathematical settings that bear little connection to the historical developments one and a half, two or three centuries earlier. (ibid.; emphasis added)

For all his attempts to distance himself from Boyer's idolisation of the triumvirate, ${ }^{10}$ Fraser here commits himself to a position similar to Boyer's. Namely, Fraser claims that modern punctiform A-track analysis is, "of course, [!] a primary point of reference" for understanding the analysis of the past. His sentiment that modern punctiform B-track analysis bears little connection to the historical developments ignores the procedure versus ontology dichotomy.

Arguably, modern infinitesimal analysis provides better proxies for the procedures of the calculus of the founders, based as it was on a fundamental Leibnizian distinction between assignable and inassignable quantities ${ }^{11}$ underpinning the infinitesimal analysis of the 17th and 18th centuries, as analyzed in Błaszczyk et al. [17]. Meanwhile scholars since Ishiguro [55, Chapter 5] have been engaged in a syncategorematic quest for ghosts of departed quantifiers in Leibniz.

A sentiment of the inevitability of classical analysis is expressed by Fraser who feels that "classical analysis developed out of the older subject and it remains a primary point of reference for understanding the eighteenth-century theories" (ibid.); yet his very formulation involves circular reasoning. It is only if one takes classical analysis as a primary point of reference that it becomes plausible to conclude that it developed out of the older subject (and therefore should serve as a primary point of reference).

To comment on Ian Hacking's distinction between the butterfly model and the Latin model, we note the contrast between a model of a deterministic biological development of animals such as butterflies, as opposed to a model of a contingent historical evolution of languages such as Latin.

If one allows, with Ian Hacking [47, p. 72-75], for the possibility of alternative courses for the development of analysis (a Latin model as opposed to a butterfly model), Fraser's

\footnotetext{
${ }^{10}$ See note 9 on page 126 for an explanation of the term.

${ }^{11}$ The distinction goes back to the work of Nicholas of Cusa (1401-1464), which also inspired Galilio's distinction between quanta and non-quanta according to Knobloch [67].
} 
assumption that the historical development necessarily led to modern classical analysis (as formalized by Weierstrass and others) remains an unsupported hypothesis.

The butterfly model assumes a teleological or culminationist view of mathematical development, in which the texts of historical mathematicians are interpreted in terms of their location on the track that inevitably culminates in our current dominant formal understanding of analysis. In contrast, the Latin model treats some aspects of mathematical development as contingent rather than inevitable.

By distinguishing A-track from B-track development, we are not therefore proposing a butterfly model, just with two possible butterflies at the end. Rather, we are noting that it is an illusion that one can interpret historical mathematics without the interpretation being informed by philosophical commitments or mathematical frameworks. Fraser and others we have referenced in this paper have not applied a framework-free interpretation of the works of mathematicians such as Leibniz, Euler, and Cauchy. They have, consciously or unconsciously, employed an A-track or Weierstrassian framework. By focusing attention on mathematical procedure rather than ontology, we can see that a B-track framework reveals aspects of these great mathematicians' work that A-track analyses distort or dismiss. The B-track analysis also provides an alternative to the claustrophobic presumption that mathematical development is pre-scripted or determined.

We will respond to Fraser's little connection claim in more detail in Section 5.

5. Euclid. In this section we will respond more fully to Fraser's passage quoted in Section 4.6.

5.1. History and 'little connection'. Since 1870, both non-Archimedean analysis and non-Archimedean geometry have developed in parallel to classical analysis. Here one could mention the work of Otto Stolz and Paul du Bois-Reymond in analysis, and that of Giuseppe Veronese and David Hilbert in geometry; see Ehrlich [31].

Hermann Hankel developed the first modern interpretation of Book V of Euclid's Elements in his 1876 work [48]. In this way he initiated mathematical investigations of the notion of magnitude carried out further by Stolz, Du Bois-Reymond, H. Weber, and O. Hölder; for details see Błaszczyk [13].

This line of investigation culminated in 1899/1903 with the first and second editions of Grundlagen der Geometrie, when Hilbert pioneered an axiom system for an ordered field; see Hilbert [53], [54]. With his axiomatic method, Hilbert initiated a new type of investigation of a foundational type in geometry. However, Hilbert also initiated an axiomatic study of the real numbers, which is a lesser-known aspect of his work. Such new investigations focus, on the one hand, on axiomatic characterisations of mathematical structures, and on the other, on the descriptive power of formal languages.

As regards the real numbers, foundational studies were carried out, among others, by E. V. Huntington and O. Veblen, at the beginning of the 20th century. In 1926 Artin and Schreier [2] and in 1931 Tarski [99] developed the theory of real-closed fields. Robinson explicitly referred to the "theory of formally-real fields of Artin and Schreier" in [82, p. 278]. Robinson's framework for infinitesimal analysis inscribes in the tradition of foundational studies of the real numbers.

Hilbert's foundational studies in geometry are well-known. While his Grundlagen der Geometrie is primarily a mathematical book, it is referred to both by mathematicians studying the foundations of Euclidean geometry and by historians interpreting Euclid's geometry; see e.g., Heath [50], Mueller [76]. Historians of Greek mathematics often rely on modern 
interpretive techniques. Thus Netz [78] refers to cognitive science and Kuhn's philosophy. Naturally also refer to the achievements of modern mathematics.

Now, a given historian's competence and the limitations of the mathematical techniques he learned, tend to determine what portion of contemporary mathematics he is able to apply in his historical studies. ${ }^{12}$ This applies even to the history of Greek mathematics, where the distance between the source text we refer to and mathematical techniques we apply in our interpretation is measured in thousands of years, as compared to hundreds in the case of infinitesimal calculus. In the next two sections we will provide some examples.

5.2. Applying modern mathematics to interpreting Euclid. The very first proposition of Euclid's Elements is controversial as it relies on the assumption that two circles occurring in its proof must meet. Almost every commentator from Proclus to Mueller comments on this assumption. Already in the 1645 Claude Richard [81] realized that an additional hypothesis is required here; see de Risi ([30, Section 1.2]).

5.2.1. Hilbert's axioms. Heath claimed that one "must invoke" the continuity axiom to fill this logical gap in Euclid's proof [50, vol. 1, p. 242]. However, interpretaters of Euclid's Elements following Hilbert's Grundlagen der Geometrie showed that in place of the continuity axiom, it is sufficient to invoke the so-called Pasch axiom and circle-circle intersection property, and then Euclid's geometry, as developed in Books 1-4, can be developed in a plane without the continuity axiom; see Hartshorne [49, p. 110].

Furthermore, the Pasch axiom can be derived from the fact that the field of segments is a Pythagorean field, i.e., a field closed under the operation $\sqrt{1+a^{2}}$; see [49, p. 187].

5.2.2. Modern axiomatic systems. Modern axiomatic systems that followed, do not appeal explicitly to the notion of a circle. Instead, they encode the circle-circle intersection property in some axioms which apply relations of congruence, betweenness, and equal distance. Thus, Karol Borsuk and Wanda Szmielew modified Hilbert axiom system, and showed that Euclid's geometry can be developed based on axioms of incidence, congruence, equal distance and order, including the Pasch axiom, without the continuity axiom; see [21, $\S 93]$.

5.2.3. Tarski's axioms. In 1959 Alfred Tarski [100] obtained further results using a system of axioms he developed. This system can be formulated in first order logic and enables one to translate some metageometrical results into ordered field terms. Thus, it turns out that models of Euclidean geometry coincide (up to isomorphism) with Cartesian planes over a Euclidean field, i.e., an ordered field closed under the square root operation.

In 1974 Szmielew [97] showed that models of geometry satisfying the Pasch axiom coincide (up to isomorphism) with a Cartesian plane over a Pythagorean field. The so called twocircles axiom ${ }^{13}$ provides the geometric analog for the Euclidean field property. Furthermore,

1. the Pasch axiom is a consequence of the circle axiom;

2. the Pasch axiom does not follow from the continuity axiom, and

\footnotetext{
${ }^{12}$ Such limitations are presumably what prompts a historian to describe a given piece of mathematics as abstruse; see Section 4.6 and Section 1.

${ }^{13}$ The circle axiom asserts that $B(a b c) \Rightarrow\left(\exists c^{\prime}\right)\left[B\left(p b c^{\prime}\right) \wedge D\left(a c^{\prime} a c\right)\right]$. The two circles axiom asserts that
}

$$
M\left(a c^{\prime} b\right) \wedge M\left(a b^{\prime} c\right) \wedge M\left(b a^{\prime} c\right) \Rightarrow(\exists q)\left[L\left(p c^{\prime} q\right) \wedge\left(D\left(q b^{\prime} a b^{\prime}\right) \vee D\left(q a^{\prime} b a^{\prime}\right)\right)\right],
$$

where $M(a b c)$ means that $b$ is the midpoint, i.e., lies between $a$ and $c$, and $D(a b b c)$ while $L$ is the colinearity relation, $B$ is the betweenness relation, and $D$ is the equal distance relation. 
3. the circle axiom indeed follows from the continuity axiom;

see respectively Szmielew [95], Szmielew [96], and Szczerba [94]. With regard to this somewhat paradoxical situation, Szmielew commented as follows: "At the first glance the situation seem to be paradoxical, since it is common to say that $\mathrm{C}$ [the circle axiom] is a particular consequence of $\mathrm{Co}$ [the continuity axiom]. In fact, $\mathrm{C}$ can not be proved without the use of $\mathrm{Co}$, however, in the proof of $\mathrm{C}$ besides Co also some other axioms of $\mathrm{E}$ [Euclidean geometry] are involved" [96, p. 751].

Relating modern axioms, on the one hand, to Euclid's axioms, postulates and theorems, on the other, constitutes a methodological challenge since that there is no explicit counterpart of betweenness relation in Euclid's geometry. Nonetheless, such metageometrical results arguably contribute both to our interpretation and appreciation of Euclid's Elements and to the book's historical perception, by enabling new interpretive perspectives, be it logical gaps in Euclid's development or the role of diagrams in his proofs. This is contrary to Fraser's contention that analysis of historical texts that relies on modern mathematics cannot produce a significant contribution to history. While this methodology is widely accepted, it differs from the one we apply only in the scope of modern mathematics being employed.

5.3. Applying modern mathematics to the real numbers. Our next example concerns the real numbers. In light of the foundational studies mentioned above, we have today not only different versions of the continuity axiom but also some independence results. We know, for example, that continuity of real numbers can be characterized by the Dedekind axiom (i.e., no Dedekind cut gives a gap) or equivalently by the conjunction of two conditions: Cauchy completeness (CC) and the Archimedean axiom (AA). It is known that axioms $\mathrm{CC}$ and $\mathrm{AA}$ are independent, since both the hyperreals and Levi-Civita fields are nonArchimedean but Cauchy-complete.

With this knowledge we now consider the historical texts. In 1872 neither Cantor, Dedekind, Heine, or Méray were aware of AA. However, while Dedekind characterized the real numbers by his axiom (and from the current perspective it is a correct characterisation), Cantor, as well as Méray and Heine, characterized real numbers by means of CC alone. Can we then claim that they (i.e., Cantor, Heine, and Méray) really knew what real numbers are? Apparently not.

Furthermore, while Cauchy sequences, or Fundamentalreihen, were widely in use at the time, Dedekind's axiom by no means bears any connection to historical developments that preceded Stetigkeit und irrationale Zahlen.

To give a further example, the Intermediate Value Theorem (IVT) is another equivalent form of the the Dedekind Cut Axiom; see Teismann [101, section 3], and Błaszczyk [14, note 5]. This relates to the famous Bolzano proof of the IVT [19]. Bolzano had no access to axiomatic characterisations of the real numbers. Should this modern result be employed in analyzing Bolzano's pamphlet? We argue that the answer is affirmative, as this result suggests a new interpretive perspective of seeking another version of the continuity axiom that he implicitly used in his proof.

As far as Robinson's framework is concerned, we have shown that, from a historical perspective, it belongs squarely in the tradition of foundational studies of the real numbers. However, Robinson's framework also sheds new light on the relation of being infinitely close, as well as on infinite (or in modern terminology, hyperfinite) sums and products (see Section 6.6). Therefore Robinson's framework can be usefully applied in historiography on a par with Hilbert's axiomatic method and the results it provides, beyond the strictures of 
a Weierstrassian framework useful though it may be.

6. From general to the specific. In addition to general claims concerning an alleged a priori inapplicability of Bernoullian frameworks to historical research as analyzed in Section 4, Fraser's article contains some specific claims concerning Robinson's framework. We will analyze one such claim in this section.

6.1. Algorithms. As we mentioned in Section 4, Fraser himself implicitly relies on the dichotomy of procedure versus ontology. Thus, he writes:

Two of the most prominent features of the early calculus - the tension between analytic and geometric modes of representation and the central place occupied by the algorithm - are not reproduced at all in nonstandard analysis as defining characteristics of the subject. [40, p. 39] (emphasis added)

We will examine the "tensions" mentioned by Fraser on another occasion. What interests us here is Fraser's claim concerning "the central place occupied by the algorithm." Now algorithmic aspects clearly fall under the rubric of procedures as opposed to ontology (see Section 2.2). Focus on algorithms certainly characterized 18th century mathematics. We will examine Fraser's claim that they allegedly "are not reproduced at all in nonstandard analysis" in Section 6.3.

6.2. Fraser, algebraic analysis, and the details. Fraser argues that infinitesimals and the familiar foundational issues surrounding them were not the primary motivators of the evolution of analysis. He further claims that the bulk of 19th-century work responded to the context of the algebraic-analytic approach exemplified by Lagrange, in which infinitesimals already played a secondary role:

The question of the logical status of infinitesimals is of secondary interest, from either the perspective of a researcher in the early eighteenth century or an observer today. The second topic concerns the decisive shift to algebraic analysis that occurred in the writings of such figures as Euler and Lagrange in the second half of the century. [40, p. 28] (emphasis added)

Fraser continues:

The French philosopher Auguste Comte ... revered Lagrange and believed that he had brought mathematics to an almost completed state... In this conception questions about the status of infinitesimals or the meaning of imaginary numbers were very much secondary, and the primary emphasis was on operations, functions, relations and the active process of working to solutions. [40, p. 37]

While initially plausible, this thesis dissolves under closer scrutiny of the actual details. The plausibility of Fraser's thesis hinges on equivocation on the meaning of the term algebraic used by Fraser. What exactly is meant by Lagrange's algebraic approach? One can envision the following three possibilities for the meaning of this term:

1. Lagrange's approach to function theory based on power series expansion;

2. the principle of the so-called generality of algebra extensively relied upon by Lagrange following his predecessors;

3. the algebraic foundations for analysis as initiated in Euler's Introductio [33]. 
Item (1) will not do since Cauchy extensively deals with nonanalytic functions. Thus, (1) is not plausible as a general description of 19th century work, since it leaves out Cauchy, who certainly was an important figure for 19th century analysis.

Item (2), rather than being the context of Cauchy's work, was on the contrary the main source of his dissatisfaction with Lagrange, as detailed in the introduction to Cauchy's Cours d'Analyse [23].

This leaves us with (3) Euler's Introductio and subsequent work by Lagrange and others. Fraser's assumption of a clean separation between algebra and infinitesimals may be compatible with modern usage, but it is an anachronistic way of reading Euler's work where algebraic manipulations with infinitesimals were ubiquitous; for details see Bascelli et al. [8]. In fact, Lagrange himself eventually came to embrace infinitesimals in the second edition of his Mécanique Analytique; see Katz-Katz [59].

Thus, Euler's work can be viewed as an algebraic approach dealing with algebraic manipulations of infinitesimal and non-infinitesimal entities alike. Hence (3) is not a compelling argument that infinitesimals only played a minor role. The actual details are not kind to Fraser's thesis.

6.3. Fraser, hyperreal analysis, and the details. Formulating assertions concerning a mathematical theory would require that a scholar possess a basic level of competence with regard to the theory, particularly if he seeks to base sweeping historical conclusions on such claims. In this section we will examine the level of Fraser's competence in the matter of the hyperreals.

In his discussion of the hyperreals, Fraser finally provides some details while attempting to summarize Robinson's construction of the hyperreals via the compactness theorem applied to a suitable language $K$.

What is alarming is that Fraser [40, p. 25] specifically describes his collection $K$ as consisting of sentences of the form $c<v$ for each $c \in \mathbb{R}$ (and no other sentences) in order to force an infinite $v$ in the model. Earlier in that paragraph, Fraser does comment on a "formalized language that is rich enough to formulate all sentences that are true in the real numbers $\mathbb{R}$," but this comment is not used in any way in his definition of $K$ (and furthermore contains a crucial inaccuracy: transfer applies to first-order sentences only, not to all sentences as Fraser claims). Now any proper ordered field extension of $\mathbb{R}$ will satisfy the sentences of Fraser's collection $K$, for example the field of rational functions ordered by the behavior of the function at infinity (i.e., a suitable lexicographic order).

Neither the compactness theorem nor hifalutin' mathematical logic are required to build a proper ordered field extension of $\mathbb{R}$. Thus Fraser entirely misses the point of the hyperreals as the only extension possessing a full transfer principle (see Section 1). The transfer principle connects procedurally to Leibniz's Law of continuity. Leibniz's theoretical strategy in dealing with infinitesimals was analyzed in Katz-Sherry [65], Sherry-Katz [88], Bascelli et al. [8], Błaszczyk et al. [16], as well as the study by Bair et al. [3] that Fraser is reacting to. Leibniz's theoretical strategy was arguably more robust than George Berkeley's flawed critique thereof.

We therefore cannot agree with Fraser's claim to the effect that Leibniz allegedly "never developed a coherent theoretical strategy to deal with [infinitesimals]" in [40, p. 31]. Fraser's attitude toward Leibniz's theoretical strategy in dealing with infinitesimals is surely a function of his dismissive attitude toward B-track historiography in general, which would similarly account for a failure to appreciate the import of Robinson's extension. 
6.4. Transfer principle. The transfer principle is a type of theorem that, depending on the context, asserts that rules, laws or procedures valid for a certain number system, still apply (i.e., are "transfered") to an extended number system. Thus, the familiar extension $\mathbb{Q} \hookrightarrow \mathbb{R}$ preserves the property of being an ordered field. To give a negative example, the extension $\mathbb{R} \hookrightarrow \mathbb{R} \cup\{ \pm \infty\}$ of the real numbers to the so-called extended reals does not preserve the property of being an ordered field. The hyperreal extension $\mathbb{R} \hookrightarrow{ }^{*} \mathbb{R}$ preserves all first-order properties, e.g., the formula $\sin ^{2} x+\cos ^{2} x=1$ (valid for all hyperreal $x$, including infinitesimal and infinite values of $x \in{ }^{*} \mathbb{R}$ ). A construction of such an extension $\mathbb{R} \hookrightarrow{ }^{*} \mathbb{R}$ appears in Section 1. For a more detailed discussion, see Keisler's textbook Elementary Calculus [66].

6.5. Does calculus become algorithmic over the hyperreals? Contrary to Fraser's claim cited in Section 1, algorithms indeed play a central role in analysis exploiting the extension $\mathbb{R} \hookrightarrow{ }^{*} \mathbb{R}$. A typical example is the definition of the differential ratio $\frac{d y}{d x}$ for a plane curve as

$$
\operatorname{st}\left(\frac{\Delta y}{\Delta x}\right)
$$

where $\Delta x$ is an infinitesimal $x$-increment and $\Delta y$ is the corresponding change in the variable $y$. Here "st" is the standard part function which rounds off each finite hyperreal number to the nearest real number. Thus, Robinson's framework precisely provides an algorithm for computing the differential ratio, which is procedurally similar to Leibniz's transcendental law of homogeneity; see Katz-Sherry [65] and Sherry-Katz [88] for an analysis of the primary sources in Leibniz.

This modern B-track procedure is closer to the historical methods of the pioneers of the calculus than to the (mathematically equivalent) modern A-track procedures. This is because the latter involve a non-constructive notion of limit, where the value $L$ of the limit cannot be given algorithmically by a formula comparable to (1), but rather has to be given in advance, so that one can then elaborate an Epsilontik proof that $L$ is the correct value. In this sense, the B-track procedure as summarized in (1) provides a better proxy for Leibniz's algorithm than the A-track procedure, contrary to Fraser's claim. For an analysis of further constructive and algorithmic aspects of the hyperreal framework see Sanders [83], [84]. [85].

6.6. Euler's procedures and their proxies. Another example illustrating the advantage of B-track over A-track when seeking proxies for the procedures of mathematical analysis as it was practiced historically is Euler's definition of the exponential function. Fraser repeatedly mentions non-Archimedean analysis and non-Archimedean fields in his article, but it is not entirely clear whether he understands that the hyperreals are not merely another non-Archimedean field. In any such field there are infinitesimals and infinitely large numbers incorporated in the structure of an ordered field. However, over the hyperreals one has further structures, such as the theory of infinite, or more precisely hyperfinite, sums like $a_{1}+\ldots+a_{N}$ where $N$ is an infinite hyperinteger, as well as infinite products, which provide proxies for Euler's procedures where A-track proxies are not available; see Bair et al. [4]. Thus we can follow Euler's procedure in writing the exponential function $e^{k z}$ as

$$
\left(1+\frac{k z}{i}\right)^{i}
$$

where $i$ is infinite number, in [33] vol. I, p. 93, namely an infinite product. We can also follow Euler when he develops such a product into an infinite sum using the binomial formula (for an 
infinite exponent!), where A-track historians see only 'dreadful foundations' (see Section 1).

Euler's application of the binomial formula with an infinite exponent is rewritten by Ferraro [35, p. 48] in modern $\sum_{r=0}^{\infty}$ notation relying on a Weierstrassian notion of limit ("for every $\varepsilon>0$ there is an $N$ such that if $n>N$ then the partial sum satisfies $\left|L-\sum_{i=0}^{n} \ldots\right|<\varepsilon$, etc.") Yet Euler's procedure here admits a closer proxy in terms of a hyperfinite sum with $i+1$ terms, where $i$ is an infinite hyperinteger; see Bair et al. [4, p. 222] for a discussion. A related analysis of a reductionist reading of Euler by H. M. Edwards appears in Kanovei et al. [58].

6.7. Surreal blunder. The surreal number field was developed by Conway [29] or alternatively by Alling [1]. This field provides an example of a B-continuum that possesses no transfer principle. ${ }^{14}$

Conway's maximal class surreal field is denoted No. Ehrlich [32] refers to it as the absolute arithmetic continuum. This term has the correct connotations since the surreal system is of some pertinence specifically in arithmetic, being the absolutely maximal number system one could possibly develop. This may one day make the Guinness Book of Records, but the field No is only marginally relevant in analysis, since even a simple function like the sine function cannot be extended to all of No. Conway's system therefore cannot serve as a foundation for analysis since $N o$ does not satisfy transfer beyond being a real closed field.

Namely, there is no surreally defined predicate of being an integer which still honors transfer. Conway's own version of surintegers fail to satisfy transfer since $\sqrt{2}$ turns out to be surrational.

On the other hand, Alling's surreals are field-isomorphic to a properly defined hyperreal field with whatever transfer one wishes. Thus the surreals do admit extensions of the usual relations of being natural, etc., and the usual functions like the sine function, but only via the hyperreals, rather than by means of the sur-construction.

Meanwhile, [40, p. 26] lists the surreals as one of the possible frameworks for nonArchimedean analysis, alongside the hyperreal framework and Bell's framework for Smooth Infinitesimal Analysis. But, as noted by Ehrlich [32, Theorem 20], the field No is isomorphic to a maximal (class) hyperreal field, and therefore cannot be said to be an independent framework, especially since any transfer principle in the surreals necessarily derives from the hyperreals. Including the surreals on par with these viable frameworks for analysis is a misjudgment that puts into question Fraser's technical ability to evaluate the appropriateness of modern mathematical frameworks for interpreting the procedures of the mathematics of the past.

7. Demonisation of Laugwitz and Euler. In this section we analyze Fraser's attitude toward the scholarship of Detlef Laugwitz. It turns out that Fraser repeatedly misrepresents Laugwitz's work. Fraser did this on at least two occasions: in his 2008 article for the New Dictionary of Scientific Biography, and in his latest piece in 2015 for the monograph Delicate Balance. The latter term can only be applied with difficulty to his one-sided presentation of the state of Cauchy scholarship today.

7.1. Laugwitz's scholarship on analysis in Cauchy. In the abstract of his 1987 article in Historia Mathematica, Laugwitz is careful to note that he interprets Cauchy's sum theorem "with his [i.e., Cauchy's] own concepts":

\footnotetext{
${ }^{14}$ See Section 6.4 for a brief introduction to the transfer principle.
} 
It is shown that the famous so-called errors of Cauchy are correct theorems when interpreted with his own concepts. [70, p. 258]

In the same abstract, Laugwitz goes on to stress that

No assumptions on uniformity or on nonstandard numbers are needed. (emphasis added)

Laugwitz proceeds to give a lucid discussion of the sum theorem in terms of Cauchy's infinitesimals in Section 7 on pages 264-266, with not a whiff of modern number systems. In particular this section does not mention the article Schmieden-Laugwitz [86]. In a final section 15 entitled "Attempts toward theories of infinitesimals," Laugwitz presents a general discussion of how one might formalize Cauchyan infinitesimals in modern set-theoretic terms. A reference to the article by Schmieden and Laugwitz appears in this final section only. Thus, Laugwitz carefully distinguishes between his analysis of Cauchy's procedures, on the one hand, and the ontological issues of possible implementations of infinitesimals in a set-theoretic context, on the other.

7.2. Fraser's assumptions. Alas, all of Laugwitz's precautions went for naught. In 2008, he became a target of damaging innuendo in the updated version of The Dictionary of Scientific Biography. Here Fraser writes as follows in his 2008 article on Cauchy:

Laugwitz's thesis is that certain of Cauchy's results that were criticized by later mathematicians are in fact valid if one is willing to accept certain assumptions about Cauchy's understanding and use of infinitesimals. These assumptions reflect a theory of analysis and infinitesimals that was worked out by Laugwitz and ... Schmieden during the 1950s. [39, p. 76] (emphasis added)

Fraser claims that Laugwitz's interpretation of Cauchy depends on assumptions that reflect a modern theory of infinitesimals. Fraser's indictment, based on the Omega-theory of Schmieden and Laugwitz (see e.g., [86]), is off the mark, as we showed in Section 1. In the intervening years Fraser has apparently not bothered to read Laugwitz's article [70] either. Indeed, Fraser's verdict is unchanged seven years later in 2015:

Laugwitz, ... some two decades following the publication by Schmieden and him of the $\Omega$-calculus[,] commenced to publish a series of articles arguing that their nonArchimedean formulation of analysis is well suited to interpret Cauchy's results on series and integrals. [40, p. 27]

What Fraser fails to mention is that Laugwitz specifically and explicitly separated his analysis of Cauchy's procedures from attempts to account ontologically for Cauchy's infinitesimals in modern terms, as we showed in Section 1.

Fraser's dual strategy in his article involves praising his allies and undermining his opponents. He seeks to present an allegedly united front of received A-tracker scholarship on Cauchy, while distancing himself from Boyer whose bowing down to the triumvirate ${ }^{15}$ even Fraser finds it difficult to defend. Fraser also adopts ad hominem arguments when it comes to dealing with his opponents such as Laugwitz, by suggesting that their goal in interpreting history involves an inappropriate interposition of a lens of their professional preoccupations in non-Archimedean mathematics.

\footnotetext{
$\overline{{ }^{15} \text { See note } 9}$ on page 126 for an explanation of the term.
} 
Fraser's first line of attack, involving an allegedly united Epsilontik front, is as questionable as the second. Cracks in triumvirate unity are ubiquitous. Thus, Schubring speaks with surprising frankness about his disagreement with Grabiner's ideas on Cauchy-Weierstrass:

I am criticizing historiographical approaches like that of Judith Grabiner where one sees epsilon-delta already realized in Cauchy [87, Section 3]

(see also Błaszczyk et al. [18]). Fraser similarly overplays his hand when he quotes GrattanGuinness's distinction between history and heritage in [46]. For it is precisely in his 2004 article that Grattan-Guinness called explicitly for a re-evaluation of Cauchy, by putting into question the Epsilontik track, and warns against reading Cauchy as if he had read Weierstrass already:

The (post-)Weierstrassian refinements have become standard fare, and are incorporated into the heritage of Cauchy; but it is mere feedback-style ahistory to read Cauchy (and contemporaries such as Bernard Bolzano) as if they had read Weierstrass already ... On the contrary, their own pre-Weierstrassian muddles need historical reconstruction, and clearly [46, p. 176] (emphasis added).

It is precisely Fraser's Cauchy-Weierstrass line that is being referred to as heritage (rather than history) here. Grattan-Guinness also wrote that Cauchy's proof of the sum theorem is difficult to interpret because it is stated in infinitesimal terms (see Section 4.5), acknowledging the limitations of the A-track approach when it comes to interpreting Cauchy's 1853 text [28] on the summation of series. Weierstrass' followers broke with Cauchy's infinitesimal mathematics, but one will not discover this by reading Fraser's comments on Cauchy.

A curious aspect of Fraser's text is his sweeping claim against the relevance of modern non-Archimedean theories to the history of analysis. Fraser's misunderstandings arguably arise from failing to distinguish between procedure and ontology. He fails to cite some of the key studies of Euler, such as Kanovei [56] and McKinzie-Tuckey [75]; Laugwitz's article [70] is cited but not discussed, and instead misrepresented by Fraser (see Section 1); Laugwitz's article [71] appears in Fraser's bibliography but is not mentioned in the body of Fraser's article.

7.3. Gray on Euler's foundations. The relevance of modern B-track theoretical frameworks can be established by the fact that the routine A-tracker claims that Euler's foundations are allegedly dreadful can today be discarded in favor of a far greater appreciation of the coherence of his infinitesimal techniques (see e.g., Section 6.6). Thus, Euler's foundations are described as dreadfully weak by Jeremy Gray:

Euler's attempts at explaining the foundations of calculus in terms of differentials, which are and are not zero, are dreadfully weak. [44, p. 6]

Such sweeping pronouncements come at a high price in anachronism. Characteristically, Gray does not provide any justification for such a claim. Such were indeed the received views prior to the work of Robinson, Laugwitz, Kanovei, and others.

7.4. Shaky arguments. In a similar vein, Judith Grabiner talks about shaky arguments and unerring intuition:

... eighteenth-century mathematicians had an almost unerring intuition. Though they were not guided by rigorous definitions, they nevertheless had a deep understanding of the properties of the basic concepts of analysis. This conclusion is 
supported by the fact that many apparently shaky eighteenth-century arguments can be salvaged, etc. [43, p. 358] (emphasis added)

This passage of Grabiner's sheds no light on how Euler and other 18th century mathematicians may have hit upon such unerring intuitions to guide their work in infinitesimal analysis, in the first place. Short of an account of mathematical intuition, unerring intuition does almost no explanatory work. It merely tells us that Euler, Lagrange, and others had a special power. To be sure, there are theories of mathematical intuition; those of Kant and Brouwer come to mind. But neither of those theories is primarily concerned with a power for making mathematical discoveries.

The coherence of the procedures of an 18th century master like Euler is better understood in light of their modern proxies within consistent theories of infinitesimals, making Euler's arguments appear less shaky than those of Fraser, Grabiner, and Gray.

7.5. Summary of conclusions. We have examined Fraser's stated position and analyzed the assumptions underpinning his text [40]. A summary of our conclusions follows.

7.5.1. Fraser claims that classical analysis, understood as modern Weierstrassian analysis, is a "primary point of reference" for the historiography of analysis, for the stated reason that it "developed out of the older subject." However, Fraser's claim involves circular reasoning, since his inference depends on the assumption of a teleological view of the history of analysis (as having the Weierstrassian framework as its inevitable goal), criticized by Ian Hacking in terms of the dichotomy of the butterfly model and the Latin model for the evolution of mathematics.

7.5.2. By adhering to the teleological view, Fraser commits himself to Boyer's position while paying lip service to a critique of Boyer's subservience to "the great triumvirate" and arithmetisation.

7.5.3. Fraser's claim that Leibniz allegedly "never developed a coherent theoretical strategy to deal with [infinitesimals]" reveals his lack of familiarity with recent literature published in Erkenntnis, Studia Leibnitiana, and HOPOS, elaborating the details of just such a strategy of Leibniz's; see Katz-Sherry [65], Sherry-Katz [88], Bascelli et al. [8].

7.5.4. Fraser claims that no "significant contribution to history" can result from applying modern theories to the history of analysis, but he appears to make an exception for modern Archimedean theories as developed by Weierstrass and his followers, as we showed in the context of a thought experiment involving (non-) Archimedean issues in Section 2.1.

7.5.5. The Grabiner-Fraser so-called "Cauchy-Weierstrass" foundation was and remains a tale (for details see Borovik-Katz [20], Katz-Katz [60], Bascelli et al. [10]), or more specifically an A-tracker ideological commitment, rather than an accurate historical category.

7.5.6. Fraser's claim that "Robinson and Lakatos were mistaken in their assessment of Cauchy" is a non-sequitur exacerbated by Fraser's failure to make explicit the alleged mistakes of Robinson and Lakatos.

7.5.7. Fraser wishes to place "Cauchy within a given intellectual and historical milieu" but in point of fact yanks Cauchy right out of his milieu and insert him in a 1870 milieu.

7.5.8. Fraser cites Grattan-Guinness on mathematical history versus mathematical heritage, but by Gratan-Guinness's own standard with regard to Cauchy, it is Fraser himself who indulges in mathematical heritage at the expense of history. 
7.5.9. Fraser's perhaps most fundamental philosophical blunder is his failure to appreciate the importance of the distinction of procedure versus ontology when it comes to interpreting the mathematics of the past.

8. Construction of the hyperreals. Since certain historians find hyperreal numbers to be abstruse (see Section 4.6), we include a construction of a hyperreal field to demonstrate that it requires no more background than a serious undergraduate course in algebra including the theorem on the existence of a maximal ideal.

In an approach to analysis exploiting Robinson's framework, one works with the pair $\mathbb{R} \subseteq$ ${ }^{*} \mathbb{R}$ where $\mathbb{R}$ is the usual ordered complete Archimedean continuum, whereas ${ }^{*} \mathbb{R}$ is a proper extension thereof. Such a field ${ }^{*} \mathbb{R}$ could be called a Bernoullian continuum, in honor of Johann Bernoulli who was the first systematically to use an infinitesimal-enriched continuum as the foundation for analysis. Robinson's field ${ }^{*} \mathbb{R}$ obeys the transfer principle (see Section 1).

A field ${ }^{*} \mathbb{R}$ can be constructed from $\mathbb{R}$ using sequences of real numbers. To motivate the construction, it is helpfpul to analyze first the construction of $\mathbb{R}$ itself using sequences of rational numbers. Let $\mathbb{Q}_{C}^{\mathbb{N}}$ denote the ring of Cauchy sequences of rational numbers. Then

$$
\mathbb{R}=\mathbb{Q}_{C}^{\mathbb{N}} / \operatorname{MAX}
$$

where "MAX" is the maximal ideal in $\mathbb{Q}_{C}^{\mathbb{N}}$ consisting of all null sequences (i.e., sequences tending to zero).

The construction of a Bernoullian field can be viewed as a relaxing, or refining, of the construction of the reals via Cauchy sequences of rationals. This can be motivated by a discussion of rates of convergence as follows. In the above construction, a real number $u$ is represented by a Cauchy sequence $\left\langle u_{n}: n \in \mathbb{N}\right\rangle$ of rationals. But the passage from $\left\langle u_{n}\right\rangle$ to $u$ in this construction sacrifices too much information. We seek to retain a bit of the information about the sequence, such as its "speed of convergence." This is what one means by "relaxing" or "refining" the equivalence relation in the construction of the reals from sequences of rationals.

When such an additional piece of information is retained, two different sequences, say $\left\langle u_{n}\right\rangle$ and $\left\langle u_{n}^{\prime}\right\rangle$, may both converge to $u \in \mathbb{R}$, but at different speeds. The corresponding "numbers" will differ from $u$ by distinct infinitesimals. For example, if $\left\langle u_{n}\right\rangle$ converges to $u$ faster than $\left\langle u_{n}^{\prime}\right\rangle$, then the corresponding infinitesimal will be smaller. The retaining of such additional information allows one to distinguish between the equivalence class of $\left\langle u_{n}\right\rangle$ and that of $\left\langle u_{n}^{\prime}\right\rangle$ and therefore obtain distinct hyperreals infinitely close to $u$. Thus, the sequence $\left\langle\frac{1}{n^{2}}\right\rangle$ generates a smaller infinitesimal than $\left\langle\frac{1}{n}\right\rangle$.

A formal implementation of the ideas sketched above is as follows. Let us outline a construction of a hyperreal field ${ }^{*} \mathbb{R}$. Let $\mathbb{R}^{\mathbb{N}}$ denote the ring of sequences of real numbers, with arithmetic operations defined termwise. Then we have

$$
{ }^{*} \mathbb{R}=\mathbb{R}^{\mathbb{N}} / \mathrm{MAX}
$$

where "MAX" is a suitable maximal ideal of the $\operatorname{ring} \mathbb{R}^{\mathbb{N}}$. What we wish to emphasize is the formal analogy between (2) and (3). ${ }^{16}$

We now describe a construction of such a maximal ideal MAX $=\mathrm{MAX}_{\xi}$ in terms of a finitely additive measure $\xi$. The ideal MAX consists of all "negligible" sequences $\left\langle u_{n}\right\rangle$, i.e., sequences which vanish for a set of indices of full measure, namely,

$$
\xi\left(\left\{n \in \mathbb{N}: u_{n}=0\right\}\right)=1 .
$$

\footnotetext{
${ }^{16}$ In both cases, the subfield is embedded in the superfield by means of constant sequences.
} 
Here $\xi: \mathcal{P}(\mathbb{N}) \rightarrow\{0,1\}$ (thus $\xi$ takes only two values, 0 and 1 ) is a finitely additive measure taking the value 1 on each cofinite set, ${ }^{17}$ where $\mathcal{P}(\mathbb{N})$ is the set of subsets of $\mathbb{N}$. The subset $\mathcal{F}_{\xi} \subseteq \mathcal{P}(\mathbb{N})$ consisting of sets of full measure is called a free ultrafilter. These originate with Tarski [98]. The construction of a Bernoullian continuum outlined above was therefore not available prior to that date.

The construction outlined above is known as an ultrapower construction. The first such construction appeared in [52], as did the term hyper-real. The transfer principle ${ }^{18}$ for this extension is an immediate consequence of the theorem of Łoś [73]. For a recent application in differential geometry, see Nowik-Katz [79]. For a survey of approaches to Robinson's framework, see Fletcher et al. [38].

9. Conclusion. We have exploited the dichotomy of A-track versus B-track methods in the historical development of infinitesimal calculus in analyzing the work of the great historical authors, including Leibniz, Euler, and Cauchy. We have paid attention also to the dichotomy of practice versus ontology. The historiography of mathematics as practiced by historians whose training was limited to a Weierstrassian framework falls short of the target when analyzing many of the great historical authors, due to its failure to attend to the dichotomies mentioned, as exemplified by work of Lützen and Fraser.

While we are sympathetic to Barabashev's sentiment that "as a result of the elaboration of non-standard analysis, Leibniz's discovery is differentiated more and more from Newton's theory of fluxions and fluents" [6, p. 38], we feel that a more convincing case involves the relative advantages of Robinson's framework in interpreting the historical infinitesimal analysis as compared to a Weierstrassian framework.

We have followed Ian Hacking's proposal to approach the history of mathematics in terms of a Latin (nondeterministic) model of development rather than a butterfly (deterministic) model. Carl Boyer's account of the historical development of the calculus is a classic example of a deterministic model. While in preliminary declarations, Fraser seeks to distance himself from Boyer's view of the history of analysis as inevitable progress toward infinitesimal-frei arithmetisation, actually, as we show, he commits himself to a position similar to Boyer's.

We have argued in favor of applying modern mathematical techniques beyond Weierstrass, specifically, foundational studies both in geometry and arithmetic of real numbers to analyze Euclid's Elements and 19th century constructions of real numbers. We have argued against Fraser's claim that analysis of historical texts that relies on modern mathematics beyond Weierstrass cannot produce a significant contribution to history. Our methodological premise involves applying Robinson's framework for analysis with infinitesimals to analyzing 17-, 18- and 19th century mathematical texts, following the pioneering work of Abraham Robinson and Detlef Laugwitz.

Acknowledgments. We are grateful to V. de Risi for bringing to our attention the work by Claude Richard [81]. V. Kanovei was partially supported by the RFBR grant no. 17-0100705. M. Katz was partially supported by the Israel Science Foundation grant no. 1517/12.

\footnotetext{
${ }^{17}$ For each pair of complementary infinite subsets of $\mathbb{N}$, such a measure $\xi$ "decides" in a coherent way which one is "negligible" (i.e., of measure 0) and which is "dominant" (measure 1).

${ }^{18}$ See Section 6.4 on page 133 for an explanation of the term.
} 


\section{REFERENCES}

1. Alling N., Conway's field of surreal numbers, Trans. Amer. Math. Soc., 287 (1985), no.1, 365-386.

2. Artin E., Schreier O., Algebraische Konstruktion reeller Körper., Abhandlungen aus dem Mathematischen Seminar der Hamburgischen Univeristät, Leipzig 5 (1926), p. 85-99. Reprinted in Serge Lang and John T. Tate (editors), The Collected Papers of Emil Artin, Addison-Wesley Publishing Company, Reeding, MA., 1965, p. 258-272.

3. Bair J., Błaszczyk P., Ely R., Henry V., Kanovei V., Katz K., Katz M., Kutateladze S., McGaffey T., Schaps D., Sherry D., Shnider S., Is mathematical history written by the victors? Notices of the American Mathematical Society, 60, no.7, 886-904.

4. Bair J., Błaszczyk P., Ely R., Henry V., Kanovei V., Katz K., Katz M., Kutateladze S., McGaffey T., Reeder P., Schaps D., Sherry D., Shnider S., Interpreting the infinitesimal mathematics of Leibniz and Euler, Journal for General Philosophy of Science, 48 (2017), no.2, 195-238.

5. Bair J., Katz M., Sherry D., Fermat's dilemma: Why did he keep mum on infinitesimals? and the European theological context, Foundations of Science (2018), to appear.

6. Barabashev A., In support of significant modernization of original mathematical texts (in defense of presentism), Philos. Math. (3), 5 (1997), no.1, 21-41.

7. Bascelli T., Bottazzi E., Herzberg F., Kanovei V., Katz K., Katz M., Nowik T., Sherry D., Shnider S., Fermat, Leibniz, Euler, and the gang: The true history of the concepts of limit and shadow, Notices of the American Mathematical Society, 61 (2014), no.8, 848-864.

8. Bascelli T., Błaszczyk P., Kanovei V., Katz K., Katz M., Schaps D., Sherry D., Leibniz vs Ishiguro: Closing a quarter-century of syncategoremania HOPOS: Journal of the Internatonal Society for the History of Philosophy of Science, 6 (2016), no.1, 117-147.

9. Bascelli T., Błaszczyk P., Kanovei V., Katz K., Katz M., Kutateladze S., Nowik T., Schaps D., Sherry D., Gregory's sixth operation Foundations of Science, see https://arxiv.org/abs/1612.05944

10. Bascelli T., Błaszczyk P., Borovik A., Kanovei V., Katz K., Katz M., Kutateladze S., McGaffey T., Schaps D., Sherry D., Cauchy's infinitesimals, his sum theorem, and foundational paradigms, Foundations of Science (2018), see https://arxiv.org/abs/1704.07723

11. Benacerraf P., What numbers could not be The Philosophical Review, 74 (1965), 47-73.

12. Blåsjö V., On what has been called Leibniz's rigorous foundation of infinitesimal geometry by means of Riemannian sum, Historia Math., 44 (2017), no.2, 134-149.

13. Błaszczyk P., A note on Otto Hölder's memoir; Die Axiome der Quantität und die Lehre vom Mass, Annales Universitatis Paedagogicae Cracoviensis Studia ad Didacticam Mathematicae Pertinentia, V (2013), 129-142.

14. Błaszczyk P., A purely algebraic proof of the fundamental theorem of algebra, Annales Universitatis Paedagogicae Cracoviensis Studia ad Didacticam Mathematicae Pertinentia, VIII (2016), 5-21.

15. Błaszczyk P., Katz M., Sherry D., Ten misconceptions from the history of analysis and their debunking Foundations of Science, 18 (2013), no.1, 43-74.

16. Błaszczyk P., Kanovei V., Katz K., Katz M., Kudryk T., Mormann T., Sherry D., Is Leibnizian calculus embeddable in first order logic? Foundations of Science, 22 (2017), no.4, 717-731, see http://arxiv.org/abs/1605.03501.

17. Błaszczyk P., Kanovei V., Katz K., Katz M., Kutateladze S., Sherry D., Toward a history of mathematics focused on procedures Foundations of Science, 22 (2017), no.4, 763-783, see http://arxiv.org/abs/1609.04531.

18. Błaszczyk P., Kanovei V., Katz M., Sherry D., Controversies in the foundations of analysis: Comments on Schubring's Conflicts, Foundations of Science, 22 (2017), no.1, 125-140.

19. Bolzano B. Purely analytic proof of the theorem that between any two values which give results of opposite signs, there lies at least one real root of the equation, in S. Russ (ed.), The Mathematical Works of Bernard Bolzano, Oxford University Press, Oxford, 2004, 251-263.

20. Borovik A., Katz M., Who gave you the Cauchy-Weierstrass tale? The dual history of rigorous calculus, Foundations of Science, 17 (2012), no.3, 245-276.

21. Borsuk K., Szmielew W., Foundations of geometry: Euclidean and Bolyai-Lobachevskian geometry, projective geometry. Revised English translation. North-Holland Publishing, Amsterdam, Interscience Publishers, New York, 1960. 
22. Boyer C., The concepts of the calculus, Hafner Publishing, 1949.

23. Cauchy A.L., Cours d'Analyse de L'École Royale Polytechnique. Première Partie. Analyse algébrique, Imprimérie Royale, Paris, 1821.

24. Cauchy A.L., Résumé des Leçons données à l'École Royale Polytechnique sur le Calcul Infinitésimal, Paris, Imprimérie Royale, 1823.

25. Cauchy A.L. Recherches sur l'équilibre et le mouvement intérieur des corps solides ou fluides, élastiques ou non élastiques, Bulletin de la Société philomatique, 9-13, 1823. Reprinted in Oeuvres complètes, Série 2, V.2, 300-304.

26. Cauchy A.L. "Memoire sur la rectification des courbes et la quadrature des surfaces courbes." Paris. Reprinted as Cauchy [27], (1832).

27. Cauchy A.L., Memoire sur la rectification des courbes et la quadrature des surfaces courbes, Mem. Acad. Sci. Paris, 22 (1850), 3-15. Reprinted in Oeuvres complètes, 1/II, Paris: Gauthier-Villars, (1908), 167-177.

28. Cauchy A.L., Note sur les séries convergentes dont les divers termes sont des fonctions continues d'une variable réelle ou imaginaire, entre des limites données, Reprinted in Oeuvres complètes, Series 1, Paris: Gauthier-Villars, 12 (1990), 30-36.

29. Conway J. On numbers and games, London Mathematical Society Monographs, No. 6. Academic Press [Harcourt Brace Jovanovich, Publishers], London-New York, 1976.

30. De Risi V., The Development of Euclidean Axiomatics. The systems of principles and the foundations of mathematics in editions of the Elements from Antiquity to the Eighteenth Century, Archive for History of Exact Sciences, 70 (2016), no.6, 591-676.

31. Ehrlich P., The rise of non-Archimedean mathematics and the roots of a misconception. I. The emergence of non-Archimedean systems of magnitudes, Archive for History of Exact Sciences, 60, (2006), no.1, $1-121$.

32. Ehrlich P., The absolute arithmetic continuum and the unification of all numbers great and small, Bull. Symbolic Logic, 18 (2012), no.1, 1-45.

33. Euler L., Introductio in Analysin Infinitorum, Tomus primus, SPb and Lausana, 1748.

34. Euler L., Introduction to analysis of the infinite. Book I, Translated from the Latin and with an introduction by J. Blanton. Springer-Verlag, New York, 1988 (translation of Euler [33]).

35. Ferraro G., Differentials and differential coefficients in the Eulerian foundations of the calculus, Historia Mathematica, 31 (2004), no.1, 34-61.

36. Ferraro G., Panza M., Lagrange's theory of analytical functions and his ideal of purity of method, Archive for History of Exact Sciences, 66 (2012), no.2, 95-197.

37. Fisher G. Cauchy and the infinitely small, Historia Math, 5 (1978), no.3, 313-331.

38. Fletcher P., Hrbacek K., Kanovei V., Katz M., Lobry C., Sanders S., Approaches to analysis with infinitesimals following Robinson, Nelson, and others, Real Analysis Exchange, 42 (2017), no.2, 193252.

39. Fraser C., Cauchy, In the New Dictionary of Scientific Biography, Scribners and Sons, 2 (2008), 75-79.

40. Fraser C., Nonstandard analysis, infinitesimals, and the history of calculus, A Delicate Balance: Global Perspectives on Innovation and Tradition in the History of Mathematics, D. Row \& W. Horng, eds., Birkhäuser, Springer, 2015, 25-49.

41. Fried M., The discipline of history and the 'Modern consensus in the historiography of mathematics, J. Humanist. Math., 4 (2014), no.2, 124-136.

42. Gilain C., Cauchy et le cours d'analyse de l'Ecole polytechnique, With an editorial preface by Emmanuel Grison. Bull. Soc. Amis Bibl. École Polytech., (1989), no.5.

43. Grabiner J., Is mathematical truth time-dependent?, Amer. Math. Monthly, 81 (1974), 354-365.

44. Gray J. A short life of Euler, BSHM Bulletin, 23 (2008), no.1, 1-12.

45. Grattan-Guinness I., The development of the foundations of mathematical analysis from Euler to Riemann, The MIT Press, Cambridge, Mass, London, 1970.

46. Grattan-Guinness I. The Mathematics of the Past: Distinguishing its History from our Heritage, Historia Mathematica, 31 (2004), 163-185.

47. Hacking I., Why is there philosophy of mathematics at all? Cambridge University Press, Cambridge, 2014.

48. Hankel H., Zur Geschichte der Mathematik in Alterthum und Mittelalter, Teubner, Leipzig, 1876.

49. Hartshorne R., Geometry: Euclid and beyond, Undergraduate Texts in Mathematics, Springer-Verlag, New York, 2000. 
50. Heath T., Euclid. The Thirteen Books of The Elements, V.1-3, Dover, New York 1956 (reprint of the edition Cambridge University Press, Cambridge 1926, first edition 1908).

51. Hesse M.B., Forces and fields: The concept of action at a distance in the history of physics, Dover, Mineola, NY, 1962.

52. Hewitt E. Rings of real-valued continuous functions. I, Trans. Amer. Math. Soc., 64 (1948), 45-99.

53. Hilbert D., Grundlagen der Geometrie, Festschrift zur Feier der Enthüllung des Gauss-Weber Denkmals in Göttingen, Teubner, Leipzig, 1899.

54. Hilbert D., Grundlagen der Geometrie, Teubner, Leipzig, 1903.

55. Ishiguro H., Leibniz's philosophy of logic and language. Second edition, Cambridge University Press, Cambridge, 1990.

56. Kanovei V. The correctness of Euler's method for the factorization of the sine function into an infinite product, Russian Mathematical Surveys, 43 (1988), 65-94.

57. Kanovei V., Katz K., Katz M., Mormann T., What makes a theory of infinitesimals useful? A view by Klein and Fraenkel, Journal of Humanistic Mathematics, 8 (2018), no.1 (to appear).

58. Kanovei V., Katz K., Katz M., Sherry D., Euler's lute and Edwards' oud., The Mathematical Intelligencer, 37 (2015), no.4, 48-51.

59. Katz K., Katz M., Cauchy's continuum., Perspectives on Science, 19 (2011), no.4, 426-452.

60. Katz K., Katz M., A Burgessian critique of nominalistic tendencies in contemporary mathematics and its historiography, Foundations of Science, 17 (2012), no.1, 51-89.

61. Katz K., Katz M., Stevin numbers and reality, Foundations of Science, 17 (2012), no.2, 109-123.

62. Katz M., Review of Fried [41], 2015, see http://www.ams.org/mathscinet-getitem?mr=3245163.

63. Katz M., Polev M., From Pythagoreans and Weierstrassians to true infinitesimal calculus, Journal of Humanistic Mathematics, 7 (2017), no.1, 87-104.

64. Katz M., Schaps D., Shnider S., Almost Equal: The Method of Adequality from Diophantus to Fermat and Beyond, Perspectives on Science, 21 (2013), no.3, 283-324.

65. Katz M., Sherry D., Leibniz's infinitesimals: Their fictionality, their modern implementations, and their foes from Berkeley to Russell and beyond., Erkenntnis, 78 (2013), no.3, 571-625.

66. Keisler H.J., Elementary Calculus: An Infinitesimal Approach. Second Edition, Prindle, Weber \& Schimidt, Boston, 1986.

67. Knobloch E., Galileo and Leibniz: different approaches to infinity, Archive for History of Exact Sciences, 54 (1999), no.2, 87-99.

68. Knobloch E., Leibniz's rigorous foundation of infinitesimal geometry by means of Riemannian sums, Foundations of the formal sciences, 1 (Berlin, 1999), Synthese, 133 (2002), no.1-2, 59-73.

69. Lakatos I., History of Science and Its Rational Reconstructions, In PSA 1970. Boston Studies in the Philosophy of Science, vol. viii, edited by Roger C. Buck and Robert S. Cohen, 91-108. Dordrecht: D. Reidel, 1971.

70. Laugwitz D., Infinitely small quantities in Cauchy's textbooks, Historia Mathematica, 14 (1987), 258274 .

71. Laugwitz D., Definite Values of Infinite Sums: Aspects of the Foundations of Infinitesimal Analysis around 1820, Archive for the History of Exact Sciences, 39 (1989), 195-245.

72. Lawvere F.W., Foundations and applications: axiomatization and education, New programs and open problems in the foundation of mathematics, (Paris, 2000), Bull. Symbolic Logic 9 (2003), no.2, 213-224.

73. Łoś J., Quelques remarques, théorèmes et problèmes sur les classes définissables d'algèbres, Mathematical interpretation of formal systems, 98-113, North-Holland Publishing, Amsterdam, 1955.

74. Lützen J., The foundation of analysis in the 19th century, Chapter 6, A history of analysis, 155-195, H. Jahnke (ed.), History of Mathematics, 24, Amer. Math. Soc., Providence, RI, 2003.

75. McKinzie M., Tuckey C., Hidden lemmas in Euler's summation of the reciprocals of the squares, Archive for History of Exact Sciences, 51 (1997), no.1, 29-57.

76. Mueller I., Philosophy of Mathematics and Deductive Structure in Euclid's Elements, Dover, New York, 2006 (reprint of the edition MIT Press, Cambridge, Massachusetts 1981).

77. Nakane M., Did Weierstrass's differential calculus have a limit-avoiding character? His definition of a limit in $\epsilon-\delta$ style, BSHM Bulletin, 29 (2014), no.1, 51-59.

78. Netz R., The Shaping of Deduction in Greek Mathematics, Cambridge University Press, Cambridge, 1999.

79. Nowik T., Katz M., Differential geometry via infinitesimal displacements, Journal of Logic and Analysis, 7 (2015), no.5, 1-44. 
80. Quine W., Ontological Relativity, The Journal of Philosophy, 65, no.7, 185-212.

81. Richard C., Euclidis elementorum geometricorum libros tredecim, Antwerp, Verdus, 1945.

82. Robinson A., Non-standard analysis, North-Holland Publishing, Amsterdam, 1966.

83. Sanders S., The computational content of nonstandard analysis, Proceedings Sixth International Workshop on Classical Logic and Computation, 24-40, Electron. Proc. Theor. Comput. Sci. (EPTCS), 213, EPTCS, 2016.

84. Sanders S., Reverse Formalism 16, Synthese (2017), Special Issue for SOTFOMIII, see https://arxiv.org/abs/1701.05066.

85. Sanders S., To be or not to be constructive, that is not the question, Indagationes Mathematicae, (2017), see http://dx.doi.org/10.1016/j.indag.2017.05.005.

86. Schmieden C., Laugwitz D., Eine Erweiterung der Infinitesimalrechnung, Mathematische Zeitschrift, 69 (1958), 1-39.

87. Schubring G., Comments on a paper on alleged misconceptions regarding the history of analysis: who has misconceptions? Foundations of Science, 21 (2016), no.3, 527-532.

88. Sherry D., Katz, M., Infinitesimals, imaginaries, ideals, and fictions, Studia Leibnitiana, 44 (2012), no.2, 166-192.

89. Sinkevich G., On history of epsilontics, Antiq. Math., 10 (2016), 183-204.

90. Skolem T., Über die Unmöglichkeit einer vollständigen Charakterisierung der Zahlenreihe mittels eines endlichen Axiomensystems, Norsk Mat. Forenings Skr., II. Ser., 1/12 (1933), 73-82.

91. Skolem T., Über die Nicht-charakterisierbarkeit der Zahlenreihe mittels endlich oder abzählbar unendlich vieler Aussagen mit ausschliesslich Zahlenvariablen, Fundamenta Mathematicae, 23 (1934), 150-161.

92. Skolem T., Peano's axioms and models of arithmetic, Mathematical interpretation of formal systems, 1-14, North-Holland Publishing Co., Amsterdam, 1955.

93. Sørensen H., Exceptions and counterexamples: understanding Abel's comment on Cauchy's theorem, Historia Math., 32 (2005), no.4, 453-480.

94. Szczerba L., Independence of Pasch's Axiom, Bulletin de l'Académie Polonaise des Sciences. Série des Sciences Mathematiques, Astronomiques et Physiques, 18 (1970), 491-498.

95. Szmielew W., A statement on two circles as the geometric analog to Euclid's field property, Bulletin de l'Académie Polonaise des Sciences. Série des Sciences Mathématiques, Astronomiques et Physiques, 18 (1970), no.7, 759-764.

96. Szmielew W., The Pasch axiom as a consequence of the circle axiom, Bulletin de l'Académie Polonaise des Sciences. Série des Sciences Mathématiques, Astronomiques et Physiques, 18 (1970), no.12, 751758.

97. Szmielew W., The role of the Pasch axiom in the foundations of the Euclidean geometry, In L. Henkin (ed). Proceedings of the Tarski Symposium. AMS, Providence, Long Island, 1974, 123-132.

98. Tarski A., Une contribution à la théorie de la mesure, Fundamenta Mathematicae, 15 (1930), 42-50.

99. Tarski A., Sur les ensembles définissables de nombres réels, Fundamenta Mathematicae, 17 (1931), 210-239.

100. Tarski A., What is elementary geometry? Henkin L., Suppes P., Tarski A. (eds.), The Axiomatic Method, With special reference to geometry and physics, Amsterdam, NHPC, 1959, 16-29.

101. Teismann H., Toward a more complete list of completeness axioms, Amer. Math. Monthly, 120 (2013), no.2, 99-114.

102. Wartofsky M., The Relation Between Philosophy of Science and History of Science, In R.S. Cohen, P.K. Feyerabend, and M.W. Wartofsky (eds.), Essays in Memory of Imre Lakatos, 717-737, Boston Studies in the Philosophy of Science XXXIX, D. Reidel Publishing, Dordrecht, Holland, 1976.

\footnotetext{
${ }^{a}$ HEC-ULG, University of Liege, 4000 Belgium

j.bair@ulg.ac.be

${ }^{\mathrm{b}}$ Institute of Mathematics, Pedagogical University of Cracow, Poland

pb@up.krakow.pl

c Department of Mathematics, University of Idaho, Moscow, Russia

ely@uidaho.edu

${ }^{\mathrm{d}}$ Department of Mathematics, University of Namur, Belgium

vhen@math.fundp.ac.be
} 
e IPPI, Moscow, and MIIT, Moscow, Russia

kanovei@rambler.ru

${ }^{\mathrm{f}}$ Department of Mathematics, Bar Ilan University, Ramat Gan, Israel

katzmik@math.biu.ac.il

g Department of Mathematics, Bar Ilan University, Ramat Gan, Israel

katzmik@macs.biu.ac.il

h Department of Mathematics, Lviv National University, Ukraine

kudryk@mail.lviv.ua

${ }^{\mathrm{i}}$ Sobolev Institute of Mathematics, Novosibirsk State University, Russia

sskut@math.nsc.ru

j Rice University, US

thomasmcgaffey@sbcglobal.net

k Department of Logic and Philosophy of Science,

University of the Basque Country UPV/EHU, Donostia San Sebastian, Spain

ylxmomot@sf.ehu.es

${ }^{1}$ Department of Classical Studies, Bar Ilan University, Ramat Gan, Israel dschaps@mail.biu.ac.il

${ }^{m}$ Department of Philosophy, Northern Arizona University, Flagstaff, US

david.sherry@nau.edu 